\title{
$\angle$ Research Square

\section{Diagnostic Value of High-resolution Ultrasound for the Evaluation of Capsular Distension in Temporomandibular Joint Effusion}

\section{Daniel Talmaceanu}

Stomestet Dental Clinic

\section{Lavinia MD}

Iuliu Hațieganu University of Medicine and Pharmacy

\section{Csaba Csutak}

Iuliu Hațieganu University of Medicine and Pharmacy

\section{Nicolae Bolog}

Phoenix Swiss Med GmbH

\section{Daniel Leucuta}

Iuliu Hațieganu University of Medicine and Pharmacy

\section{Horatiu Rotar}

Iuliu Hațieganu University of Medicine and Pharmacy

loan Tig ( $\nabla$ nelutig@yahoo.com )

University of Oradea

\section{Smaranda Buduru}

luliu Hațieganu University of Medicine and Pharmacy

\section{Research Article}

Keywords: temporomandibular joint, effusion, magnetic resonance imaging, ultrasonography

Posted Date: March 13th, 2021

DOI: https://doi.org/10.21203/rs.3.rs-284397/v1

License: (c) (i) This work is licensed under a Creative Commons Attribution 4.0 International License. Read Full License 


\section{Abstract}

Objectives. The aim of this study was to evaluate if the increased temporomandibular joint (TMJ) capsular thickness, measured by ultrasound (US), is associated with the presence of effusion, diagnosed using MRI imaging.

Methods. 102 patients with signs and symptoms of temporomandibular disorders were included in the study. Each patient underwent US and MRI examination, 1 to 7 days following clinical examination. The US was performed with an 8-40 MHz linear transducer operating at $20 \mathrm{MHz}$. MRI was performed using an $1.5 \mathrm{~T}$ MRI device. The ROC curve was analyzed to identify the optimal cut-off value for capsular distention, which can be used as an indirect sign of TMJ effusion.

Results. The capsular width values were found to be between $0.7-3.6 \mathrm{~mm}$. The best cut-off value was 2.05 $\mathrm{mm}$ with sensitivity of $55.9 \%$ and specificity of $94.7 \%(\mathrm{p}<0.05)$. The next optimal cut-off value was $1.75 \mathrm{~mm}$ with sensitivity of $67.6 \%$ and specificity of $82.4 \%$. The area under the ROC curve was $0.78(95 \% \mathrm{Cl} 0.68$, $0.87)$.

Conclusions. Ultrasound measured capsular width can be used as an indirect sign of TMJ effusion. The most accurate cut-off value obtained in this study was $2.05 \mathrm{~mm}$.

\section{Introduction}

Fluid accumulation (effusion) inside the temporomandibular joint (TMJ) is the consequence of inflammation of the synovial membrane, with excessive production of synovial fluid [1]. This can occur as a result of major trauma of the facial structures, repeated micro-traumas or different occlusal conditions [2]. Repeated microtrauma can be generated by a prolonged muscle hyperactivity (bruxism, clenching) or by mandibular orthopedic instability [2,3]. There are also general etiological factors responsible for the inflammation of the TMJ, the most common being rheumatoid arthritis, psoriatic arthritis and ankylosing spondylitis [4].

The presence of synovitis and intra-articular fluid influences the therapeutic scheme, therefore, identifying inflammatory changes is very important. From a clinical point of view, this situation is frequently associated with joint pain, although the inflammatory changes of TMJ are not always correlated with symptom severity [3].

Currently, the gold standard for the diagnosis of joint effusion is magnetic resonance imaging (MRI), which is the only examination able to reveal early phases of inflammation represented by subchondral edema [4-6]. The use of MRI is sometimes limited due to high costs and lack of availability. Therefore, the need for alternative techniques has increased. Ultrasonography (US) is reported in the literature as a simple, noninvasive, dynamic, inexpensive technique for assessing TMJ pathology, in terms of disc position, degenerative changes and effusion [7-16].

\section{Objectives}


The aim of this study was to evaluate if the increased capsular thickness, highlighted and measured by US, is associated with the presence of joint effusion, diagnosed using MRI imaging.

Another objective was to establish the critical value of high-resolution ultrasound-measured capsular distension as an indirect marker for joint effusion.

\section{Materials And Methods}

\section{Patients}

A group of 102 patients (204 TMJs) was included in this prospective study. The patients were referred to the clinic for TMJ disorders. The inclusion criteria were signs and symptoms of TMJ internal derangements, according to Research Diagnostic Criteria for Temporomandibular Disorders (RDC/TMD) [17]. Exclusion criteria were contraindications to MRI examination (claustrophobia, ferromagnetic metal carriers), patients with pure muscular disorders and patients undergoing anti-inflammatory drug treatment. Patients in which the misaligned images or distortions due to motion artefacts made the interpretation of the MRI unreliable were excluded.

Clinical examination was performed by an oral surgery specialist (with 10 years experience) and consisted of analyzing the TMJ, the masticatory and cervical muscles and the dental occlusion. The examination of all masticatory muscle groups, both endooral and exooral, was performed in order to make a differential diagnosis between a muscular and an articular disease.

Each patient underwent US and MRI examination, 1 to 7 days following clinical examination.

All methods were carried out in accordance with relevant guidelines and regulations. Informed consent was obtained from each participant included in this study. For subjects under the age of 18 , informed consent was obtained from a parent and/or legal guardian.

Ethical approval (no. 403/02.07.2015) for the study was obtained from the Ethical Committee of the University of Medicine and Pharmacy "Iuliu Hatieganu" Cluj-Napoca.

\section{MRI examination}

All the patients included in the study were evaluated using a 1.5 Tesla MRI equipment (Siemens Avanto, Siemens, Erlangen, Germany) in the same multichannel head coil. T1-weighted, T2-weighted fat-suppression and proton density (PD) sequences obtained in the oblique-sagittal and coronal planes, in closed- and opened-mouth positions, perpendicular and parallel to the long axis of the condyle were included in the aquisition protocol [18]. The MRI examination protocol is presented in Table 1. 
Table 1

MRI protocol for TMJ examination

\begin{tabular}{|c|c|c|c|c|c|c|c|c|c|}
\hline Plane & $\begin{array}{l}\text { Mouth } \\
\text { closed/open }\end{array}$ & Sequence & $\begin{array}{l}\text { TR } \\
\text { (ms) }\end{array}$ & $\begin{array}{l}\text { TE } \\
\text { (ms) }\end{array}$ & NEX & ETL & $\begin{array}{l}\text { Slice } \\
\text { thickness } \\
(\mathrm{mm})\end{array}$ & $\begin{array}{l}\text { FOV } \\
(\mathrm{mm})\end{array}$ & Matrix \\
\hline \multirow[t]{2}{*}{ Coronal } & Closed & $\begin{array}{l}\text { PD fat- } \\
\text { suppression }\end{array}$ & 2000 & 21 & 1 & 77 & 3 & 150 & $256 \times 320$ \\
\hline & & T1 & 300 & 11 & 2 & 156 & 3 & 150 & $240 \times 320$ \\
\hline \multirow{2}{*}{$\begin{array}{l}\text { Oblique- } \\
\text { sagittal }\end{array}$} & Closed & PD & 2300 & 11 & 1 & 78 & 3 & 150 & $240 \times 320$ \\
\hline & & $\begin{array}{l}\text { T2 TSE fat- } \\
\text { suppression }\end{array}$ & 4500 & 78 & 2 & 16 & 3 & 150 & $218 \times 320$ \\
\hline Coronal & Open & PD & 2000 & 20 & 1 & 77 & 3 & 150 & $256 \times 320$ \\
\hline \multirow{2}{*}{$\begin{array}{l}\text { Oblique- } \\
\text { sagittal }\end{array}$} & Open & PD & 2300 & 11 & 1 & 78 & 3 & 150 & $240 \times 320$ \\
\hline & & $\begin{array}{l}\text { T2 TSE fat- } \\
\text { suppression }\end{array}$ & 4500 & 78 & 2 & 16 & 3 & 150 & $218 \times 320$ \\
\hline $\begin{array}{l}\text { MRI - ma } \\
\text { time, TE- } \\
\text { - field of }\end{array}$ & $\begin{array}{l}\text { etic resonanc } \\
\text { ho time, NEX } \\
N\end{array}$ & $\begin{array}{l}\text { maging, TMJ J } \\
\text { number of sig }\end{array}$ & $\begin{array}{l}\text { tempc } \\
\text { l avera }\end{array}$ & $m a n$ & $\begin{array}{l}\text { ular joi } \\
\text { echo t }\end{array}$ & ain & $\begin{array}{l}\text { roton dens } \\
\text { th, TSE - T }\end{array}$ & $\begin{array}{l}\text { y, TR - } \\
\text { bo spir }\end{array}$ & $\begin{array}{l}\text { etition } \\
\text { cho, FOV }\end{array}$ \\
\hline
\end{tabular}

The presence of effusion and disc displacement were assessed in all TMJs. Joint effusion was established by identifying thin lines or areas of high signal intensity inside the articular space on T2-weighted sequences (Fig. 1b, Fig. 2b). Disc displacement was confirmed when the posterior margin of the posterior band was situated anteriorly to the vertical orientation of the condyle (the twelve o'clock line). The sideway-dislocated disc was best seen on oblique-coronal sequences and was present when the disc crossed over one of the lines through the condylar poles [19].

Synovitis is also detected as a thickening of the synovial membrane (diffused or irregular) that occurs as a T2 hypersignal and enhancement after administration of intravenous contrast medium during MRI examination. In some chronic cases, synovial fluid may appear inhomogeneous due to inside foreign bodies of a few millimeters ("rice bodies"). This is especially observed in patients with rheumatoid arthritis [20]. Usually our standard MRI protocol does not include the intravenous contrast administration and we do not routinely administer contrast in patients with TMJ disorders.

\section{The ultrasound examination}

The ultrasound examinations were performed on a Sonotouch and Tablet System (Ultrasonix Medical Corporation, Richmond, Canada) machine, with a linear transducer with a variable frequency between 8-40 $\mathrm{MHz}$. A 10 years maxillofacial ultrasonography experienced examiner evaluated all the patients, using the 20 $\mathrm{MHz}$ frequency. The transducer was placed perpendicular to the zygomatic arch and parallel with the vertical ramus of the mandible, corresponding to the anatomical location of the TMJ. The images were obtained in the transversal and longitudinal plane. The intraarticular fluid was depicted either directly by visualization of a marked hypoechoic area in the joint or by measuring the capsular width, between the superior condylar 
surface and the most lateral point of the articular capsule. All the measurements were performed in closedmouth position (Fig. 1a, Fig. 2a). The capsular width was measured in millimeters (mm). The articular bone surfaces, represented by the articular eminence and the mandibular condyle, are identified as two hyperechoic arcuate lines (Fig. 3). At US examination, the disc is described as an hyperchoic line surrounded by a hypoechoic area [21]. Normally, the disc is located between the two anatomical landmarks mentioned above (Fig. 4). The anterior or posterior position compared to normal, anatomical location, is defined as disc displacement.

Both examiners were blinded regarding the clinical examination and the other imaging results.

\section{Statistical analysis}

The receiver operator characteristic curve (ROC) was plotted for the presence of the joint effusion identified with MRI, using the capsular width ( $\mathrm{mm}$ ) as measured by $20 \mathrm{MHz}$ US. Its chart was plotted along with a $95 \%$ confidence interval computed by bootstrapping. The best cut-off was computed by identifying the best Youden index (sensitivity + specificity - 1). A table with all the cut-off values as well as with all sensitivities and specificities was computed. All statistical analysis were carried out with the R environment for statistical computing and graphics (R Foundation for Statistical Computing, Vienna, Austria), version 3.4.3 [22].

\section{Results}

The study sample consisted of 102 subjects (204 TMJs), with median age of 29 years (range 13-69 years). Of the total subjects included in the study, 84 were females.

The capsular thickness values ranged between 0.7 and $3.6 \mathrm{~mm}$ and are shown in Table 2, together with the presence/absence of joint effusion diagnosed using MRI. MRI showed effusion in $11 \%$ of the joints with normal disc position. The rest of the joints (89\%) with effusion presented disc displacement. From the total number of examined joints, 4 presented pure lateral disc displacement (Fig. 5). 
Table 2

Capsular width values and the presence/absence of joint effusion confirmed by MRI

\begin{tabular}{|c|c|c|c|}
\hline & \multicolumn{3}{|c|}{ JOINT EFFUSION - MRI } \\
\hline & YES & NO & TOTAL \\
\hline \multicolumn{4}{|c|}{ CAPSULAR THICKNESS (mm)- US } \\
\hline 0.7 & 0 & 2 & 2 \\
\hline 0.8 & 1 & 4 & 5 \\
\hline 0.9 & 0 & 7 & 7 \\
\hline 1 & 0 & 10 & 10 \\
\hline 1.1 & 1 & 18 & 19 \\
\hline 1.2 & 2 & 13 & 15 \\
\hline 1.3 & 2 & 15 & 17 \\
\hline 1.4 & 3 & 24 & 27 \\
\hline 1.5 & 0 & 14 & 14 \\
\hline 1.6 & 1 & 17 & 18 \\
\hline 1.7 & 1 & 16 & 17 \\
\hline 1.8 & 4 & 12 & 16 \\
\hline 1.9 & 0 & 5 & 5 \\
\hline 2 & 0 & 4 & 4 \\
\hline 2.1 & 3 & 3 & 6 \\
\hline 2.2 & 4 & 2 & 6 \\
\hline 2.3 & 3 & 1 & 4 \\
\hline 2.4 & 1 & 1 & 2 \\
\hline 2.5 & 1 & 1 & 2 \\
\hline 2.6 & 1 & 0 & 1 \\
\hline 2.9 & 2 & 0 & 2 \\
\hline 3 & 1 & 0 & 1 \\
\hline 3.1 & 1 & 0 & 1 \\
\hline 3.2 & 0 & 1 & 1 \\
\hline
\end{tabular}

MRI - magnetic resonance imaging, US - ultrasonography 


\begin{tabular}{|lccc|}
\hline & \multicolumn{4}{c|}{ JOINT EFFUSION - MRI } \\
\hline 3.4 & 1 & 0 & 1 \\
\hline 3.6 & 1 & 0 & 1 \\
\hline Total & 34 & 170 & 204 \\
\hline MRI - magnetic resonance imaging, US - ultrasonography \\
\hline
\end{tabular}

A ROC curve was created in order to set up the most accurate value of capsular width to distinguish between TMJs with and without effusion (Fig. 6). The area under ROC curve (AUC) was $0.78(95 \% \mathrm{Cl} 0.68,0.87)$.

The list of all cut-off values, and their corresponding sensitivities and specificities are presented in Table 3. The critical area was around $2 \mathrm{~mm}$. The best cut-off value for capsular distension was $2.05 \mathrm{~mm}$ with a sensitivity of $55.9 \%$, and a specificity of $94.7 \%$. The next best cut-off value was $1.75 \mathrm{~mm}$ with a sensitivity of $67.6 \%$ and a specificity of $82.4 \%$. 
Table 3

Capsular width cut-off value $(\mathrm{mm})$ along with their corresponding sensitivities and specificities.

\begin{tabular}{|c|c|c|c|}
\hline & Capsular width cut-off value $(\mathrm{mm})$ & Sensitivity & Specificity \\
\hline 1 & $-\operatorname{lnf}$ & 100.00 & 0.00 \\
\hline 2 & 0.75 & 100.00 & 1.20 \\
\hline 3 & 0.85 & 97.10 & 3.50 \\
\hline 4 & 0.95 & 97.10 & 7.60 \\
\hline 5 & 1.05 & 97.10 & 13.50 \\
\hline 6 & 1.15 & 94.10 & 24.10 \\
\hline 7 & 1.25 & 88.20 & 31.80 \\
\hline 8 & 1.35 & 82.40 & 40.60 \\
\hline 9 & 1.45 & 73.50 & 54.70 \\
\hline 10 & 1.55 & 73.50 & 62.90 \\
\hline 11 & 1.65 & 70.60 & 72.90 \\
\hline 12 & 1.75 & 67.60 & 82.40 \\
\hline 13 & 1.85 & 55.90 & 89.40 \\
\hline 14 & 1.95 & 55.90 & 92.40 \\
\hline 15 & 2.05 & 55.90 & 94.70 \\
\hline 16 & 2.15 & 47.10 & 96.50 \\
\hline 17 & 2.25 & 35.30 & 97.60 \\
\hline 18 & 2.35 & 26.50 & 98.20 \\
\hline 19 & 2.45 & 23.50 & 98.80 \\
\hline 20 & 2.55 & 20.60 & 99.40 \\
\hline 21 & 2.75 & 17.60 & 99.40 \\
\hline 22 & 2.95 & 11.80 & 99.40 \\
\hline 23 & 3.05 & 8.80 & 99.40 \\
\hline 24 & 3.15 & 5.90 & 99.40 \\
\hline 25 & 3.30 & 5.90 & 100.00 \\
\hline 26 & 3.50 & 2.90 & 100.00 \\
\hline 27 & Inf & 0.00 & 100.00 \\
\hline
\end{tabular}




\section{Discussions}

US is successfully used to identify inflammatory changes in large joints [23, 24]. It is a noninvasive, less expensive technique than MRI. The use of US for TMJ exploration is currently controversial in the literature [8, 26]. This is due to the fact that US is a method that strictly depends on the operator and on the frequency of the transducer that is being utilized. High frequency transducers have improved the diagnostic quality of US [27]. US is an easy technique to diagnose TMJ disc displacements. In identifying the position of the disc, there are authors which argue that US is a useful diagnostic method, while others have shown that the diagnostic accuracy of US is relatively low $[25,26,28]$.

Detection of inflammatory changes in the TMJ (synovitis, effusion) can be carried out directly with US by identifying the fluid collection as a hypoechoic area or indirectly by quantifying the degree of capsular distension. Very small differences $(0.2-0.3 \mathrm{~mm})$ in measuring capsular distension significantly influence US sensitivity and specificity as a diagnostic test for TMJ effusion [29].

MRI investigation is the reference standard in the diagnosis of inflammatory diseases of the TMJ [20]. TMJ changes that occur in inflammatory diseases have similar manifestations as in other joints: subchondral bone modifications and synovial collection with synovitis. Bone pathological changes are represented by subchondral edema, subchondral erosions and bone resorption with shape modifications of the condyle and of the temporal fossa. MRI examination is the only imaging method that can highlight subchondral edema, which is one of the most important sign in the early stages of the disease. Although not pathognomonic (it can also occur in mechanical dysfunctions), subchondral edema is the result of inflammatory osteitis that occurs in the acute phase and precedes the onset of subchondral erosions. The MRI examination shows a diffuse T2 hypersignal in the subchondral bone marrow. Early signs of TMJ inflammation, such as subchondral oedema or minimal synovial thickening, can be found in T2-weighted sequences [30].

The AUC obtained in this study was 0.78 (95\% $\mathrm{Cl} 0.68-0.87)$, indicating a good diagnostic accuracy when using US to identify capsular width as an indirect marker of fluid collection in the TMJ.

The best cut-off value of the capsular distension was $2.05 \mathrm{~mm}$, with a sensitivity of $55.9 \%$ and a specificity of $94.7 \%$. The second optimal threshold value was $1.75 \mathrm{~mm}$, with a sensitivity of $82.4 \%$ and a specificity of $67.6 \%$. Sensitivity increases at values below the optimum threshold of $2.05 \mathrm{~mm}$, while specificity increases at values above $2.05 \mathrm{~mm}$.

The optimal threshold value obtained in our study was consistent with that obtained by Manfredini et al. [31]. Bas et al. [32] identified a threshold value of capsular distension of $1.65 \mathrm{~mm}$. The differences between these values are due to the operator-dependent character of US.

The diagnostic accuracy of US in the detection of TMJ effusion has been evaluated by several authors. Manfredini et al. [31] obtained a Se of $80 \%$, while Jank et al. [14] obtained a Se of $81 \%$. Tognini et al. [33] reported a good diagnostic accuracy of US, studying the ROC curve. Melchiore et al. [7] reported a Se of $70.6 \%$. All these studies used MRI investigation as a gold standard.

Elias et al. [34] attempted to establish normal values of lateral capsular thickness. This value was between 1.4-1.6 mm. Capsular thickness may increase in the case of lateral displacements of the articular disc 
(Fig. 5). Strictly lateral discal displacements are less common [3]. In our study, we had four joints with lateral disc displacement. In these situations, it is difficult to make a differential ultrasonographic diagnosis between effusion and lateral disc displacement. However, joint pain may be a sign of differential diagnosis in such cases. Synovitis and fluid accumulation inside the TMJ are often accompanied by pain. The correlation between joint pain, capsular distension and the presence of joint fluid confirmed by MRI was made by the study of Bas et al. [32]. A positive correlation was found between pain scores and the degree of fluid accumulation confirmed by MRI. The optimal threshold value of the ultrasound-measured capsular distension obtained by this group of researchers was $1.65 \mathrm{~mm}$. The link between joint pain and the presence of fluid collection in TMJ, diagnosed with MRI imaging has been investigated by several authors, most obtaining a close link between pain and the presence of intra-articular fluid [5, 35-37]. Other authors [38, 39] concluded that there is no link between joint pain and intra-articular fluid accumulation.

The strength of this study lies in the large number of joints examined (204) and the short time in which the clinical examination, US and MRI were performed. In other studies [31-33], the interval between MRI and US was 2-3 weeks. A large chronological difference between the two investigations may influence the results of the study. Inflammatory changes may remit or, on the contrary, may intensify.

Scarce research on this topic suggests the need for conducting new studies to assess intra- and interobservational variability. It was found that for minor differences in threshold values for capsular distension, sensitivity and specificity can change substantially. These minor differences of $0.2,0.3 \mathrm{~mm}$ may be due to inter- and intra-observational variability. The evaluation of this variability is difficult to achieve only by retrospective analysis of static images. For this reason, storage and analysis of videos, that can provide more information for the examiner, is recommended.

\section{Conclusions}

According to the present study, capsular distension can be used as an indirect sign of TMJ effusion. The critical area for capsular width was around $2 \mathrm{~mm}$.

Differential ultrasonographic diagnosis between lateral disc displacement and joint effusion is difficult to be obtained. In both cases there is significant capsular distension. The presence of pain may be the clinical element of the differential diagnosis.

High-resolution US did not reach the diagnostic value of MRI in the assessment of intra-articular inflammatory changes, but it remains a dynamic, simple, and less expensive technique with good diagnostic accuracy, which can be used in case of suspicion of TMJ effusion.

\section{Abbreviations}

AUC Area under the curve

$\mathrm{Cl}$ Confidence interval

ECL Echo train length 
FOV Field of view

MRI Magnetic resonance imaging

NEX Number of signal averages

PD Proton density

RDC/TMD Research diagnostic criteria for temporomandibular disorders

ROC Receiver operator characteristic

TE Echo time

TMJ Temporomandibular joint

TR Repetition time

TSE Turbo spin echo

US Ultrasound

\section{Declarations}

\section{Author contribution:}

DT- conceptualization, study design and implementation, data collection, writing

LML- conceptualization, investigation, writing

CC- investigation, writing

NB- investigation, writing

DL- statistical analyses, data interpretation

HR- supervision, validation

IAT- visualization, supervision, validation

SB- supervision, validation, writing-review and editing

All authors reviewed the results and approved the final version of the manuscript.

\section{References}

1. Rocabado M. Clinical Atlas 1, Synovial Joint Physiology.Temporo Mandibular Joint Pain Map, CEDIME (2012). 
2. Okeson J. Bell's Orofacial Pain, seventh edition. Quintessence Publishing Co, Inc (2014).

3. Okeson J. Management of temporomandibular disorders and occlusion, eighth edition. Elsevier, Inc (2020).

4. O'Connor RC, Fawthrop F, Salha R, Sidebottom AJ. Management of the temporomandibular joint in inflammatory arthritis: Involvement of surgical procedures. Eur J Rheumatol 4(2):151-156 (2017).

5. Westesson PL, Brooks SL. Temporomandibular joint: relationship between MR evidence of effusion and the presence of pain and disk displacement. Am J Roentgenol 159: 559-563 (1992).

6. Bertram S, Rudisch A, Innerhofer K, Pumpel E, Grubwieser G, Emshoff R. Diagnosing TMJ internal derangement and osteoarthritis with magnetic resonance imaging. J Am Dent Assoc 132:753-761 (2001).

7. Melchiorre D, Calderazzi A, Maddali Bongi S et al. A comparison of ultrasonography and magnetic resonance imaging in the evaluation of temporomandibular joint involvement in rheumatoid arthritis and psoriatic arthritis. Rheumatology 42:673-676 (2003).

8. Manfredini D, Guarda-Nardini L. Ultrasonography of the temporomandibular joint: a literature review. Int J Oral Maxillofac Surg 38:1229-1236 (2009).

9. Kundu H, Basavaraj P, Kote S, Singla A, Singh S. Assessment of TMJ Disorders using ultrasonography as a diagnostic tool: a review. J Clin Diagn Res 7:3116-3120 (2013).

10. Tălmăceanu D, Lenghel LM, Bolog N et al. High-resolution ultrasonography in assessing temporomandibular joint disc position. Med Ultrason 20(1):64-70 (2018).

11. Uysal S, Kansu H, Akhan O, Kansu O. Comparison of ultrasonography with magnetic resonance imaging in the diagnosis of temporomandibular joint internal derangements: a preliminary investigation. Oral Surg Oral Med Oral Pathol Oral Radiol Endod 94:115-121 (2002).

12. TogniniF, Manfredini D, Melchiorre D, Bosco M. Comparison of ultrasonography and magnetic resonance imaging in the evaluation of temporomandibular joint disc displacement. J Oral Rehabil 32(4):248-53 (2005).

13. Emshoff R, Bertram S, Rudisch A, Gassner R. The diagnostic value of ultrasonography to determine the temporomandibular joint disk position. Oral Surg Oral Med Oral Pathol Oral Radiol Endod 84:688-696 (1997).

14. Jank S, Emshoff R, Norer B et al. Diagnostic quality of dynamic high resolution ultrasonography of the TMJ. A pilot study. Int J Oral Maxillofac Surg 34:132-137 (2005).

15. Emshoff R, Brandlmaier I, Bodner G, Rudisch A. Condylar erosion and disc displacement: detection with high-resolution ultrasonography. J Oral Maxillofac Surg 61:877-881 (2003).

16. Jank S, Rudisch A, Bodner G, Brandlmaier I, Gerhard S, Emshoff R. High resolution ultrasonography of the TMJ: helpful diagnostic approach for patients with TMJ disorders? J Craniomaxillofac Surg 29:366371 (2001).

17. Dworkin S, LeResche L. Research diagnostic criteria for temporomandibular disorders: review, criteria, examinations and specifications, critique. J Craniomandib Disord 6(4):301-355 (1992).

18. Sale H, Bryndahl F, Isberg A. Temporomandibular joints in asymptomatic and symptomatic nonpatient volunteers: a prospective 15-year follow-up clinical and MR imaging study. Radiology 267(1):183-194 
(2013).

19. Aoyama S, Kino K, Amagasa T, Sakamoto I, Omura K, Honda E, Kobayashi K, Igarashi C, Yoda TM. Clinical and magnetic resonance imaging study of unilateral sideways disc displacements of the temporomandibular joint. J Med Dent Sci 49(3):89-94 (2002).

20. O'Connor RC, Fawthrop F, Salha R, Sidebottom AJ. Management of the temporomandibular joint in inflammatory arthritis: Involvement of surgical procedures. Eur J Rheumatol 4(2):151-156 (2017).

21. Habashi H, Eran A, Blumenfeld I, Gaitini D. Dynamic high-resolution sonography compared to magnetic resonance imaging for diagnosis of temporomandibular joint disk displacement. J Ultrasound Med 34:75-82 (2015).

22. R Core Team. R: A Language and Environment for Statistical Computing [Internet]. Vienna, Austria. Available via http://www.r-project.org (2016).

23. Delle Sedie A,Riente L, lagnocco A. Ultrasound imaging for the rheumatologist. VI. Ultrasonography of the elbow, sacroiliac, parasternal, and temporomandibular joints. Clin Exp Rheumatol 24(6):617-21 (2006).

24. Bierma-Zeinstra SM, Bohnen AM, Verhaar JA, Prins A, Ginai-Karamat AZ, Lameris JS. Sonography for hip joint effusion in adults with hip pain. Ann Rheum Dis 59:178-182 (2000).

25. Manfredini D, Guarda-Nardini L. Ultrasonography of the temporomandibular joint: a literature review. Int J Oral Maxillofac Surg 38:1229-1236 (2009).

26. Kundu H, Basavaraj P, Kote S, Singla A, Singh S. Assessment of TMJ Disorders using ultrasonography as a diagnostic tool: a review. J Clin Diagn Res 7:3116-3120 (2013).

27. Talmaceanu D, Lenghel LM, Bolog N, Buduru S, Leucuta D, Horatiu R. High-resolution ultrasound imaging compared to magnetic resonance imaging for temporomandibular joint disorders: An in vivo study. Eur $\mathrm{J}$ Radiol 132:109921 (2020).

28. Uysal S, Kansu H, Akhan O, Kansu O. Comparison of ultrasonography with magnetic resonance imaging in the diagnosis of temporomandibular joint internal derangements: a preliminary investigation. Oral Surg Oral Med Oral Pathol Oral Radiol Endod 94:115-121 (2002).

29. Melchiorre D, Calderazzi A, Maddali Bongi S et al. A comparison of ultrasonography and magnetic resonance imaging in the evaluation of temporomandibular joint involvement in rheumatoid arthritis and psoriatic arthritis. Rheumatology 42:673-676 (2003).

30. Emshoff R,Brandlmaier I, Schmid C, Bertram S, Rudisch A. Bone marrow edema of the mandibular condyle related to internal derangement, osteoarthrosis, and joint effusion. J Oral Maxillofac Surg 61(1):35-40 (2003).

31. Manfredini D, Tognini F, Melchiorre D, Zampa V, Bosco M. Ultrasound assessment of increased capsular width as a predictor of temporomandibular joint effusion. Dentomaxillofac Radiol 32:359-364 (2003).

32. Bas B, Yılmaz N, Gökce E, Akan H. Ultrasoundassessment of increased capsular width in temporomandibular joint internal derangements: relationship with joint pain and magnetic resonance grading of joint effusion. Oral Surg Oral Med Oral Pathol Oral Radiol Endod 112(1):112-7 (2011).

33. Tognini F, Manfredini D, Melchiorre D, Zampa V, Bosco M. Ultrasonographic vs magnetic resonance imaging findings of temporo-mandibular joint effusion. Minerva Stomatol 52:365-370 (2003). 
34. Elias MF, Birman EG, Matsuda CK, Oliveira IRS, Jorge WA. Ultrasonographic findings in normal temporomandibular joints. Braz Oral Res 20(1):25-32 (2006).

35. Suenaga S, Nagayama K, Nagasawa T, Indo H, Majima HJ. The usefulness of diagnostic imaging for the assessment of pain symptoms in temporomandibular disorders. Jpn Dent Sci Rev 52(4):93-106 (2016).

36. Park HN, Kim KA, Koh KJ. Relationship between pain and effusion on magnetic resonance imaging in temporomandibular disorder patients. Imaging Sci Dent 44(4):293-299 (2014).

37. Sano T, Westesson PL. Magnetic resonance imaging of the temporomandibular joint. Increased T2 signal in the retrodiscal tissue of the painful joints. Oral Surg Oral Med Oral Pathol Oral radiol Endod 79:511-6 (1995).

38. OhImann B, Rammelsberg P, Henschel V, Kress B, Gabbert O, Schmitter M. Prediction of TMJ arthralgia according to clinical diagnosis and MRI findings. Int J Prosthodont 19:333-338 (2006).

39. Adame CG, Monje F, Offnoz M, Martin-Granizo R. Effusion in magnetic resonance imaging of the temporomandibular joint: a study of 123 joints. J Oral Maxillofac Surg 56:314-318 (1998).

\section{Figures}
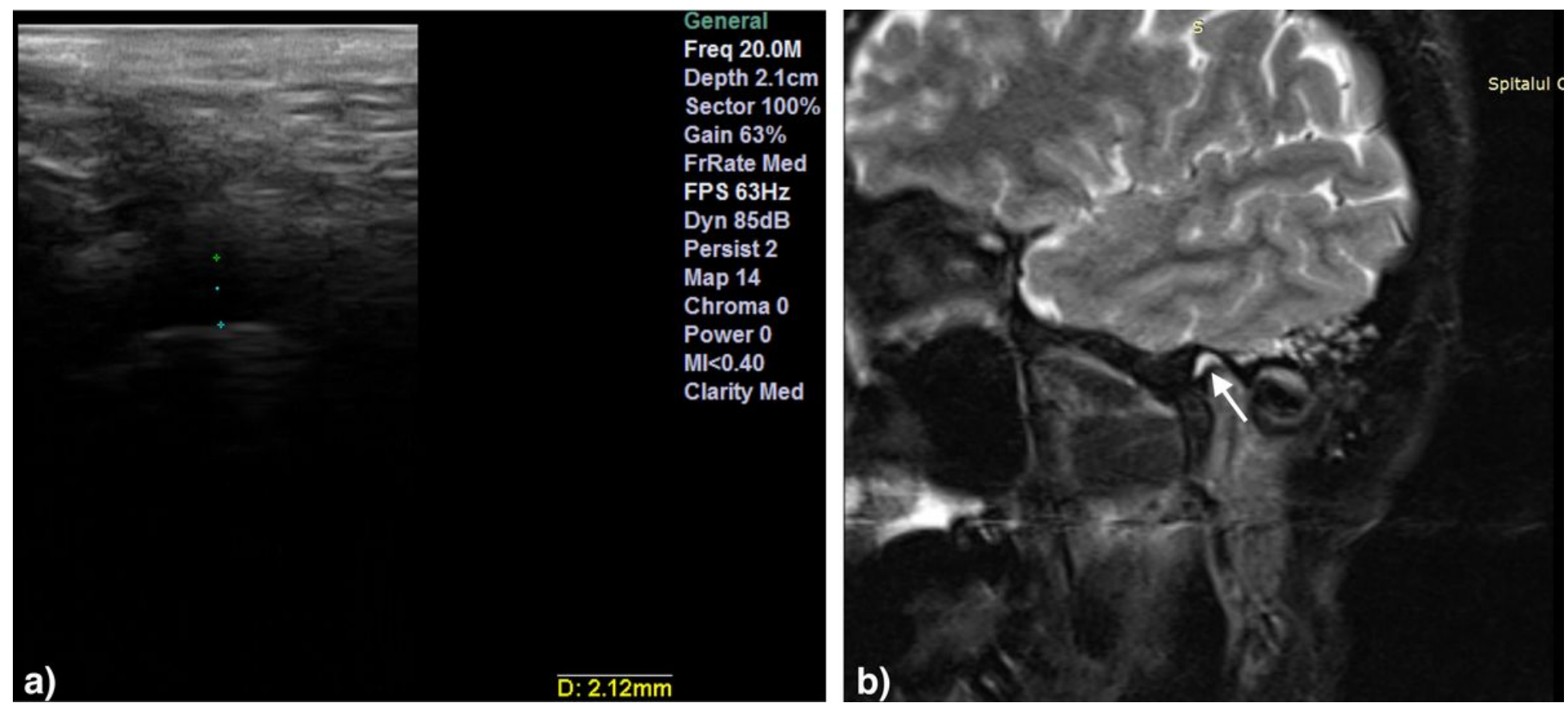

Figure 1

High-resolution $20 \mathrm{MHz}$ US image (a) of joint effusion detected by direct visualization and abnormal capsular width $(2.12 \mathrm{~mm})$. Sagittal oblique T2 weighted TSE with fat suppression image (b) of the same joint with effusion (arrow) posterior to the disc in the closed-mouth position 


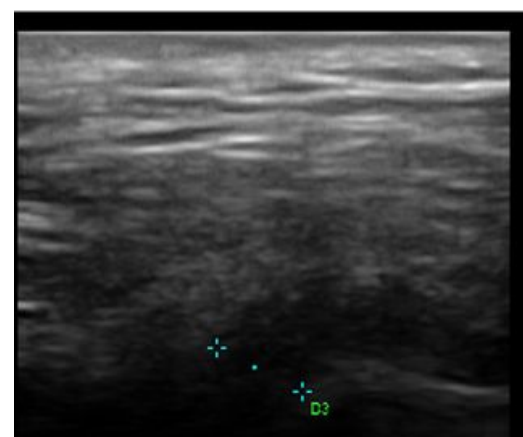

General

Freq 20.0M

Depth $2.0 \mathrm{~cm}$

Sector $100 \%$

Gain 55\%

Compound

FPS $65 \mathrm{~Hz}$

Dyn $80 \mathrm{~dB}$

Comp Low

Map 14

Chroma 0

Power 0

Ml $<0.40$

Clarity Med

\section{a)}

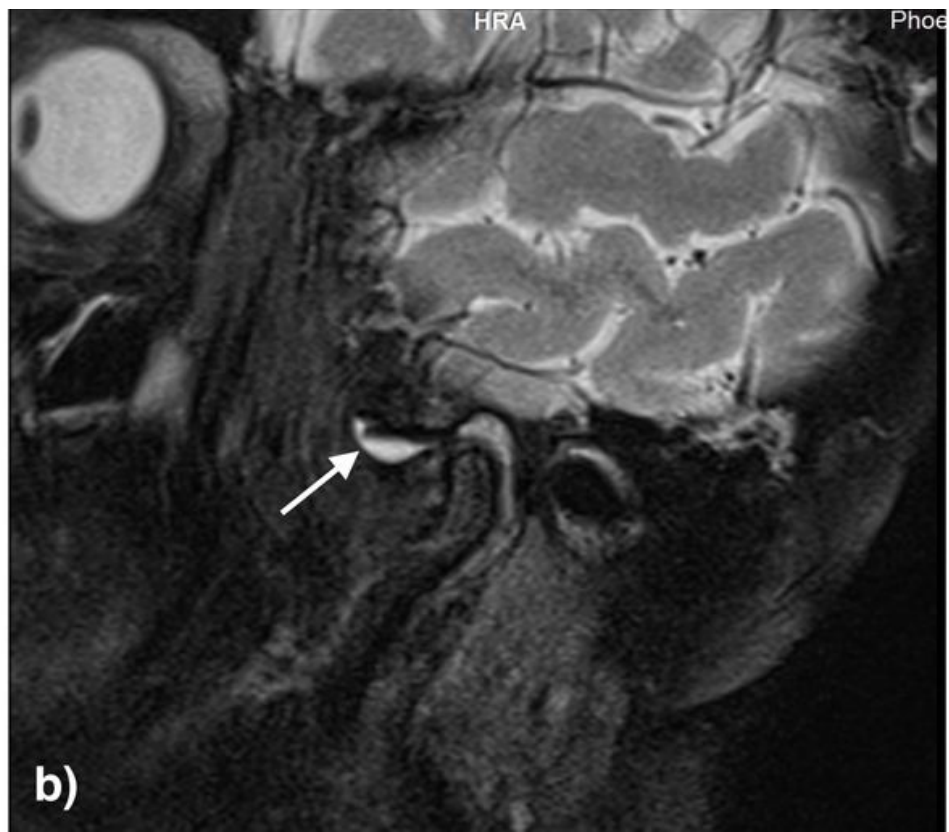

Figure 2

High-resolution $20 \mathrm{MHz}$ US image (a) of joint effusion detected by direct visualization and abnormal capsular width $(2.46 \mathrm{~mm})$. Sagittal oblique T2 weighted TSE with fat suppression image (b) of the same joint with effusion (arrow) anterior to the disc in the closed-mouth position

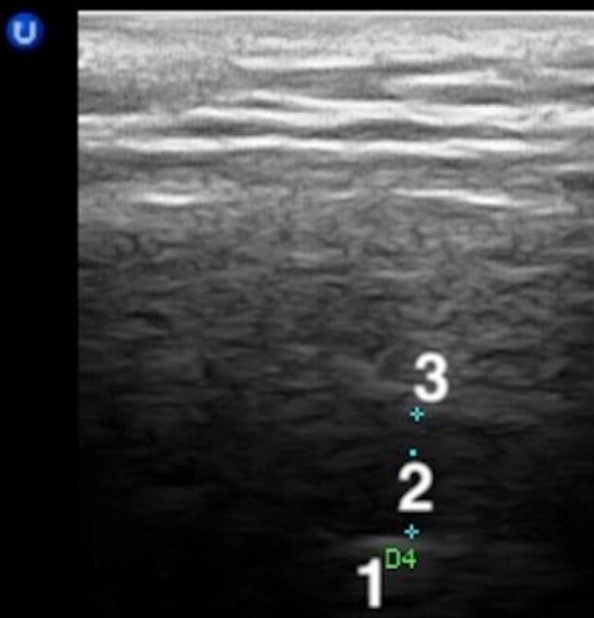

General

Freq 20.0M

Depth $3.0 \mathrm{~cm}$

Sector $100 \%$

Gain $91 \%$

FrRate Med

FPS $60 \mathrm{~Hz}$

Dyn 79dB

Persist 2

Map 14

Chroma 0

Power 0

Ml $<0.40$

Clarity Med 


\section{Figure 3}

High-resolution $20 \mathrm{MHz}$ US image of an effusion in the left TMJ detected by abnormal capsular width (2.86 mm). 1 - mandibular condyle; 2 - articular disc; 3 - glenoid fossa

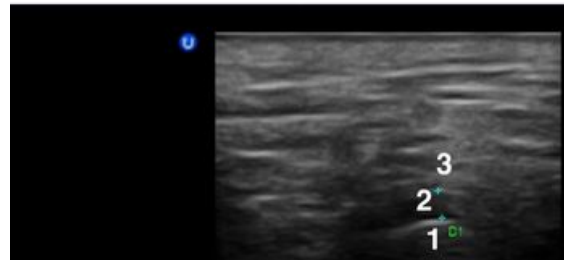

General
Freq $20.0 \mathrm{M}$
Depth $2.3 \mathrm{~cm}$
Sector $100 \%$
Gain $50 \%$
Compound
FPS $65 \mathrm{~Hz}$
Dyn $80 \mathrm{~dB}$
Comp Low
Map 14
Chroma 0
Power 0
Mk 0.40
Clarity Med

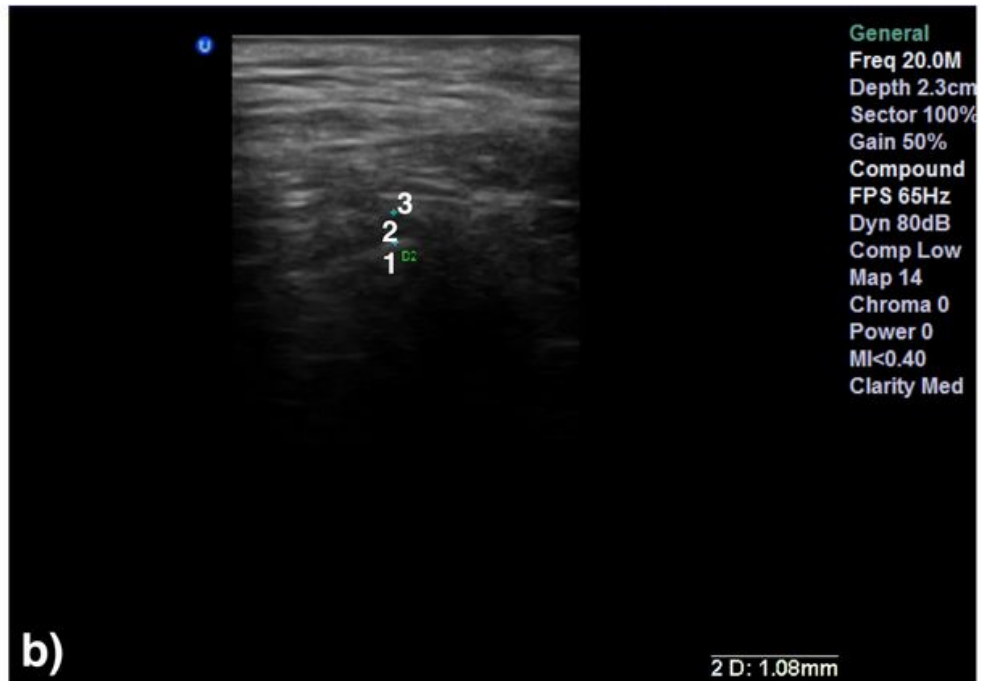

a)

\section{Figure 4}

High-resolution $20 \mathrm{MHz}$ US image of a normal TMJ: closed-mouth (a), opened-mouth (b). 1 - mandibular condyle; 2 - articular disc, situated with the intermediate part between the anterosuperior zone of the mandibular condyle and the posterosuperior part of the articular eminence; 3 - glenoid fossa
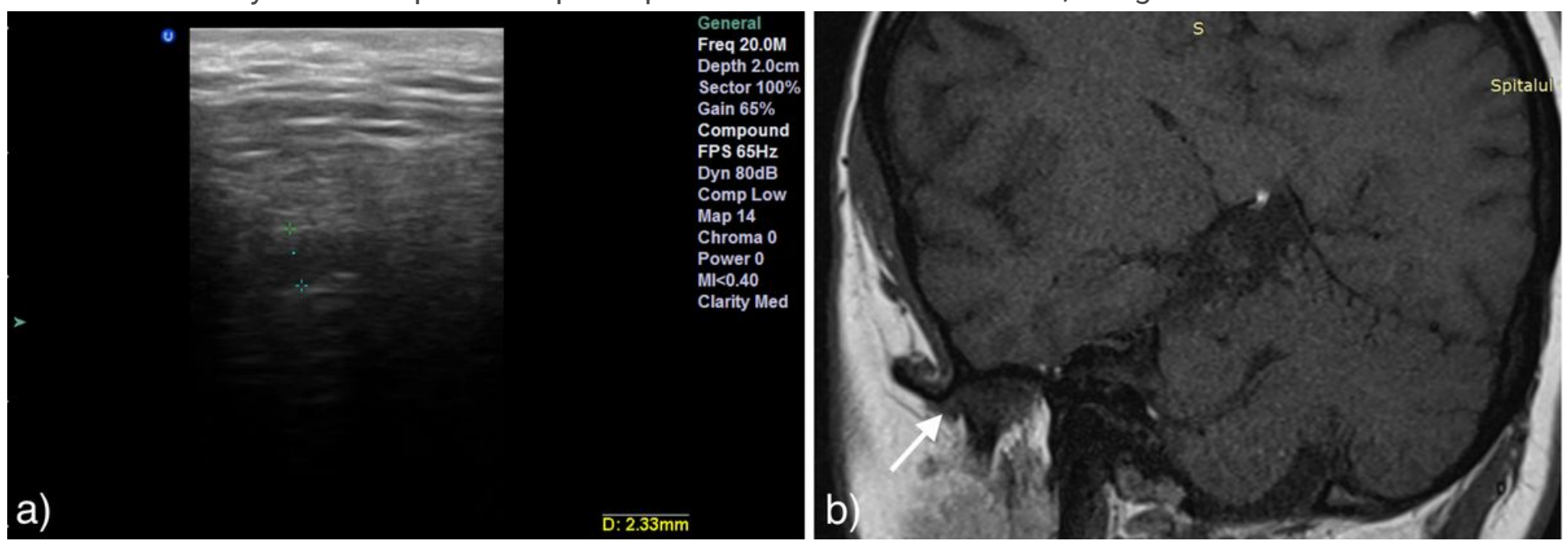

\section{Figure 5}

High-resolution $20 \mathrm{MHz}$ US image of a TMJ with abnormal capsular width $(2.33 \mathrm{~mm})$ (a). Coronal T1 closedmouth image (b) of the same TMJ with lateral disc displacement (arrow) 


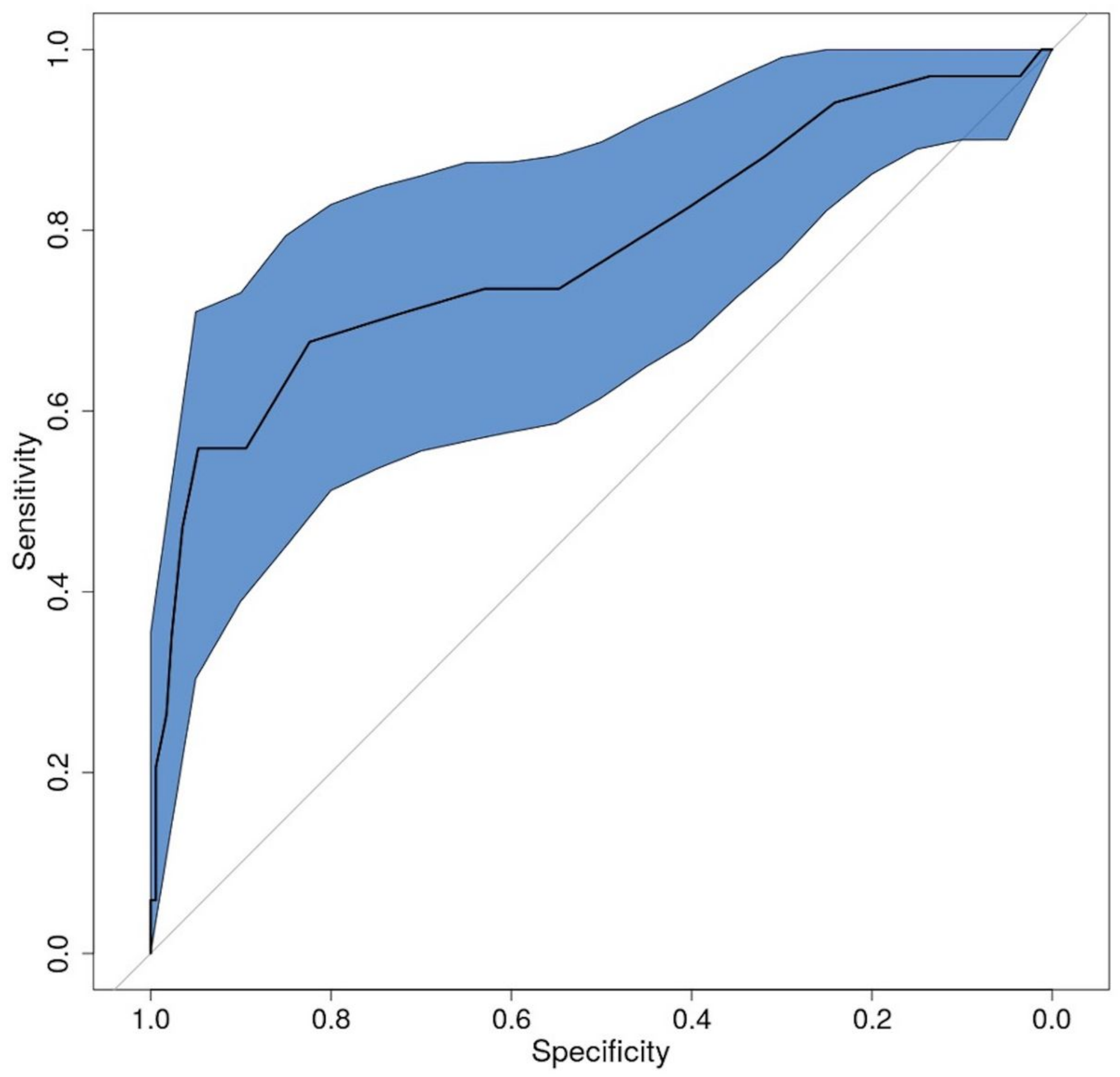

Figure 6

The receiver operator characteristic curve (ROC) was plotted for the presence of the joint fluid identified with $\mathrm{MRI}$, using the capsular width ( $\mathrm{mm}$ ) as measured by high-resolution $20 \mathrm{MHz}$ US. 